\title{
Structural Controllability of Boolean Control Networks under Partial Information
}

\author{
Qinyao Pan $\mathbb{D},{ }^{1}$ Jie Zhong $\mathbb{D},{ }^{1}$ Shalin Tong $\mathbb{D},{ }^{1}$ Bowen Li $\mathbb{D},{ }^{2,3}$ and Xiaoxu Liu $\mathbb{D}^{4}$ \\ ${ }^{1}$ College of Mathematics and Computer Science, Zhejiang Normal University, Jinhua 321004, China \\ ${ }^{2}$ School of Computer Science, Nanjing University of Posts and Telecommunications, Nanjing 210096, China \\ ${ }^{3}$ School of Information Science and Engineering, Southeast University, Nanjing 210096, China \\ ${ }^{4}$ Sino-German College of Intelligent Manufacturing, Shenzhen Technology University, Shenzhen 518000, China \\ Correspondence should be addressed to Bowen Li; qfhxjy@126.com
}

Received 23 April 2021; Accepted 20 June 2021; Published 30 June 2021

Academic Editor: Xiaodi Li

Copyright (C) 2021 Qinyao Pan et al. This is an open access article distributed under the Creative Commons Attribution License, which permits unrestricted use, distribution, and reproduction in any medium, provided the original work is properly cited.

\begin{abstract}
It is worth noting that both nodes' coupling connections and logical updating functions play a vital role in state evolutions of Boolean networks (BNs). In this paper, a new concept named structural controllability (SC) about Boolean control networks (BCNs) with known partial information on nodes' connections is studied. Then, by referring to semi-tensor product (STP) techniques, several types of SC are presented according to different issues of Boolean functions. Thereafter, several necessary and sufficient conditions are derived for SC of BCNs. Finally, a biological model of the lactose operon in Escherichia coli is simulated to show the effectiveness of the main theoretical results.
\end{abstract}

\section{Introduction}

Boolean networks (BNs) are known as discrete-time logical dynamics, firstly proposed in [1], where nodes evolute the corresponding states according to several Boolean functions. In a $\mathrm{BN}$, every gene can be chosen from a set with logical variables 1 and 0 . Therefore, a $\mathrm{BN}$ is illustrated according to certain Boolean functions $\left(g_{1}, \ldots, g_{n}\right):\{1,0\}^{n^{2}} \longrightarrow\{1,0\}^{n}$. A digraph (named as network structure digraph) with nodes $\left\{a_{1}, \ldots, a_{n}\right\}$ can well show the connections among every node (named as network structure), where there exists a directed edge from node $a_{i}$ to node $a_{j}$ if $g_{j}$ depends on $a_{i}$, i.e., if there is a sequence of variables $\widetilde{a} \in\{1,0\}^{n-1}$ satisfying $g_{j}\left(\tilde{a}, a_{i}\right) \neq g_{j}\left(\tilde{a}, a_{i}\right)$, where $\tilde{a}=\left(a_{1}, \ldots, a_{i-1}, a_{i+1}, \ldots, a_{n}\right)$. Recently, many important properties of $\mathrm{BNs}$ have been issued [2-18], and so on.

Recently, great attention has been paid to $\mathrm{BN}$ owing to a new concept called semi-tensor product (STP) of matrices [19]. STP is firstly proposed by Cheng and his collaborators [19], which is a generalization of conventional matrix product. STP can be used as an efficient tool to convert BNs into corresponding algebraic representations. Then, many fundamental results about $\mathrm{BNs}$ have been issued [20-22]. Please refer to $[23,24]$ for more applications of STP.

It is worth noting that stability and controllability are of importance in different research fields, such as impulsive differential systems [25, 26], functional differential equations [27], switched systems, and Boolean networks [28-30]. If we take external control inputs into consideration, BNs will be Boolean control networks (BCNs) established by Akutsu [28] for the first time. Note that it has been increasingly challenging for researches concerning BNs and BCNs $[29,30]$. Recently, the problem of controllability has been studied rapidly; some fundamental results have been established. To list a few, one of the main results on controllability has been issued [31], based on an input-state incidence matrix, where controllability is illustrated by the positiveness of a controllability matrix. Moreover, by Perron-Frobenius theory, controllability has been studied in [20]. In the past few years, another important issue (called pinning control method) gets great attention with less control cost and lower computational complexity [32-34]. 
It is noted that both nodes' coupling connections and logical updating functions play a vital role in state evolutions of BNs. During the past few decades, a relationship between nodes' connection and some concepts of BNs, such as stability and oscillatority, has been issued in [35-39]. Note that in real-world, not all the messages about nodes coupling and the updating Boolean functions are available, that is, only part of the nodes' connections are available. For instance, a new research concept named structural controllability (SC) concerning BCNs is well addressed in [40], whose full messages of nodes' connections are available while Boolean functions are unavailable. Therefore, it is important to analyze dynamical properties with only part of the messages on nodes' connections, i.e., all the messages of BNs are unavailable.

Therefore, based on the above analysis, some contributions of this paper are given: (i) SC of BCNs with partial information of nodes' connections available has been firstly issued, where partial nodes' connections are available; (ii) four cases of SC are defined owing to different types of network structure and logical functions. Then, several types of SC matrices are constructed using STP and Hadamard product, and some criteria are issued; (iii) SC of BCNs with partial information of nodes' connections available is a simple extension of general controllability of BCNs with full information of both network structure and logical functions.

The rest of the paper is organized as follows: in Section 2, some preliminaries are given. In Section 3, the main results of SC are given, and some criteria are obtained. In Section 4, to explain the main results, one biological model is provided. In Section 5, a brief conclusion is established.

Compared with the conference version of this paper [41], in this paper, much more detailed descriptions of the theoretical results about SC have been established. In [41], only two cases of SC are considered. However, in the revision, totally four cases of SC are discussed, and several necessary and sufficient conditions are derived for SC of BCNs, which is a generalization of the theoretical results in [41]. In addition, in this paper, some comments and detailed comparisons with the existing references have been presented, as well as a new figure has been added to better illustrate the implication relationships between four different types of SC.

Here, some notations are given: $\mathbb{N}^{+}$denotes the set consisting of positive integers. $[s: t]=\{s, s+1, \ldots, t\}$, where $s<t$ and $s, t \in \mathbb{N}^{+}$. $\delta_{n}^{m}$ is the $m$-th column of the identity matrix $I_{n} . \Delta_{n}=\left\{\delta_{n}^{m} \mid 1 \leq m \leq n\right\} . \operatorname{Col}_{k}(B)(\operatorname{Col}(B))$ denotes the $m$-th column (columns) of matrix $B . \mathfrak{R}_{q \times r}\left(\mathfrak{R}_{q \times r}\right)$ denotes the set of $q \times r$ real matrices (logical matrices). $\mathfrak{B}_{u \times v}$ is the set consisting of $u \times v$ Boolean matrices. $M=\left[\delta_{n}^{j_{1}}, \ldots, \delta_{n}^{j_{p}}\right]:=\delta_{n}\left[j_{1}, \ldots, j_{p}\right]$ is a logical matrix. Boolean addition is defined as $u+{ }_{\mathscr{B}} v:=u \vee v$, where $u, v \in \mathfrak{D} . \quad\left(A+{ }_{\mathscr{B}} B\right)_{i j}=a_{i j}+_{\mathscr{B}} b_{i j}$, where $A=\left[a_{i j}\right] \in \mathfrak{B}_{n \times s}$, $B=\left[b_{i j}\right] \in \mathfrak{B}_{n \times s}$. Blk ${ }_{i}(A)$ represents the $i$-th block of matrix $A$. A Boolean matrix satisfies all the entries belonging to $\mathfrak{D}=\{0,1\} . W_{[m, n]}=\left[I_{n} \otimes \delta_{m}^{1}, I_{n} \otimes \delta_{m}^{2}, \ldots, I_{n} \otimes \delta_{m}^{m}\right] . "$ “ $" \mathrm{de}-$ notes the known Kronecker product, “*” denotes the known Khatri-Rao product, “。” denotes the known Hadamard product, and " $<\ltimes$ " denotes STP of two given matrices.

\section{Preliminaries}

Firstly, here, some basic illustrations on STP and BCNs are introduced.

Definition 1. The STP of two given matrices $C \in \Re^{m \times n}$ and $D \in \mathfrak{R}^{p \times q}$ is defined as $C \ltimes D=\left(C \otimes I_{(s / n)}\right)\left(D \otimes I_{(s / p)}\right)$, where $s$ is the least common multiple of $n$ and $p$.

Define the bijective relationship “ $\sim$ " between D: $=\{1,0\}$ and $\Delta_{2}$ as $\gamma \sim \delta_{2}^{2-\gamma}$, which naturally extends to $\left(\gamma_{1}, \ldots, \gamma_{n}\right) \sim \ltimes_{i=1}^{n} \delta_{2}^{2-\gamma_{i}}$ between $\mathfrak{D}^{n}$ and $\Delta_{2^{\nu}}$. Equivalently, Boolean functions can be transferred into an algebraic representation.

Lemma 1. Given a logical function $\mathbf{g :} \mathfrak{D}^{n} \longrightarrow \mathfrak{D}$, its multilinear form is given as $\mathbf{g}\left(x_{1}, \ldots, x_{v}\right) \sim M_{\mathbf{g}} \sim \ltimes_{i=1}^{n} x_{i}$, where $M_{\mathbf{g}} \in \mathfrak{Q}_{2 \times 2^{v}}$ is the structure matrix, uniquely determined by $\mathbf{g}$.

Some well-known structure matrices of binary operators are presented based on Lemma 1 , such as negation "” $\left(M_{n}=\delta_{2}\left[\begin{array}{ll}2 & 1\end{array}\right]\right)$, conjunction " $\wedge$ " $\left(M_{c}=\delta_{2}\left[\begin{array}{llll}1 & 2 & 2 & 2\end{array}\right]\right)$, disjunction " $\mathrm{V}$ " $\left(M_{d}=\delta_{2}\left[\begin{array}{llll}1 & 1 & 1 & 2\end{array}\right]\right)$, conditional “ $\longrightarrow$ " $\left(M_{i}=\delta_{2}\left[\begin{array}{llll}1 & 2 & 1 & 1\end{array}\right]\right)$, and biconditional “ $\leftrightarrow$ ” $\left(M_{e}=\right.$ $\left.\delta_{2}\left[\begin{array}{llll}1 & 2 & 2 & 1\end{array}\right]\right)$.

Normally, a BCN consisting of $v$ nodes and $\kappa$ external inputs can be described:

$$
x_{i}(\tau+1)=\mathbf{g}_{i}\left(\left[x_{k}(\tau)\right]_{k \in \Theta_{i}},\left[\bar{x}_{k}(\tau)\right]_{k \in \bar{\Theta}_{i}},\left[\vartheta_{k}(\tau)\right]_{k \in \Xi_{i}},\left[\bar{\vartheta}_{k}(\tau)\right]_{k \in \bar{\Xi}_{i}}\right), \quad i \in[1: \nu]
$$

where the negation of $x$ is denoted by $\bar{x}$, i.e., $\bar{x}=x$. Here, $x_{1}, \ldots, x_{v} \in \mathfrak{D}$ express the states, $\vartheta_{i} \in \mathfrak{D}$ expresses the control input, and $\Theta_{i} \subseteq[1: \nu]$ and $\Xi_{i} \subseteq[1: \kappa]$ are the index sets of the in-neighbors of $i$-th node. For each $i \in[1: \nu]$, we call sets $\Theta_{i}, \Xi_{i}$ as the activating coupling node sets of the $i$-th node. In addition, for each $i \in[1: \nu], \bar{\Theta}_{i} \subseteq[1: \nu] \backslash \Theta_{i}$ and $\bar{\Xi}_{i} \subseteq[1: \kappa] \backslash \Xi_{i}$ are the index sets of coupling nodes of $i$-th node, and we call sets $\bar{\Theta}_{i}, \bar{\Xi}_{i}$ as the inhibiting couplings node sets of the $i$-th node. $\mathbf{g}_{i}: \mathfrak{D}^{\left|\Theta_{i}\right|+\left|\bar{\Theta}_{i}\right|+\left|\Xi_{i}\right|+\left|\bar{\Xi}_{i}\right|} \longrightarrow \mathfrak{D}, i \in[1: \nu]$, are Boolean functions, which only consist of " $\wedge$ " and " $\vee$." Therefore, the logical dynamics of (1) is only connected by logical operators “ $\wedge$," " $\vee$," and “." Thus, given a BCN (1), one can always obtain its corresponding algebraic representation using Lemma 1. 
2.1. Problem Formulation. It is noted that dynamical evolutions of BN (1) are determined by the Boolean functions $\mathbf{g}_{1}, \ldots, \mathbf{g}_{v}$, as well as its coupling sets $\Theta_{i}, \bar{\Theta}_{i}, \Xi_{i}$, and $\bar{\Xi}_{i}$, $i \in[1: \nu]$. In practical biological applications, due to experimental impacts and other reasons, we might only know the in-neighbors of some nodes, but cannot acquire the coupling relationship for each node. Therefore, assume that only partial information of (1) is known in this paper. Here, we assume that the sets $\Theta_{i}, \bar{\Theta}_{i}, \Xi_{i}$, and $\bar{\Xi}_{i}$, $i \in\left\{i_{1}, \ldots, i_{\mu}\right\} \subseteq[1: \nu]$, are given beforehand, while binary operators among different nodes in $\Theta_{i}, \bar{\Theta}_{i}, \Xi_{i}$, and $\bar{\Xi}_{i}$, $i \in\left\{i_{1}, \ldots, i_{\mu}\right\} \subseteq[1: \nu]$, are unavailable, which implies that for nodes $x_{i_{1}}, \ldots, x_{i_{\mu}}$, the corresponding activating coupling nodes, and inhibiting coupling nodes are given, whereas binary operators among nodes are unavailable.

For simple illustrations, suppose only activating coupling nodes and inhibiting coupling nodes for nodes $x_{1}, \ldots, x_{\mu}$, are given, which is as follows:

$$
\begin{cases}x_{i}(\tau+1)=\mathbf{g}_{i}\left(\left[x_{k}(\tau)\right]_{k \in \Theta_{i}},\left[\bar{x}_{k}(\tau)\right]_{k \in \bar{\Theta}_{i}},\left[\vartheta_{k}(\tau)\right]_{k \in \Xi_{i}},\left[\bar{\vartheta}_{k}(\tau)\right]_{k \in \bar{\Xi}_{i}}\right), & i \in[1: \mu], \\ x_{j}(\tau+1)=\mathbf{g}_{j}\left(\left[x_{k}(\tau)\right]_{k \in \Theta_{j}},\left[\bar{x}_{k}(\tau)\right]_{k \in \bar{\Theta}_{j}},\left[\vartheta_{k}(\tau)\right]_{k \in \Xi_{j}},\left[\bar{\vartheta}_{k}(\tau)\right]_{k \in \bar{\Xi}_{j}}\right), & j \in[\mu+1: \nu],\end{cases}
$$

where $\Theta_{i}, \bar{\Theta}_{i}, \Xi_{i}$, and $\bar{\Xi}_{i}, i \in[1: \mu]$, are known and $\Theta_{j}, \bar{\Theta}_{j}, \Xi_{j}$, and $\bar{\Xi}_{j}, j \in[\mu+1: \nu]$, are unknown. The binary operators among all the nodes are unknown, which implies that the logical operators between nodes in $\Theta_{i}, \bar{\Theta}_{i}, \Xi_{i}$, and $\bar{\Xi}_{i}, i \in[1: \nu]$, are unknown.

Based on Lemma 1, one has equivalent algebraic form of logical dynamics of (2). Given sets $\Theta_{i}, \bar{\Theta}_{i}, \Xi_{i}$, and $\bar{\Xi}_{i}$, $i \in[1: \mu]$, we make the following ordering assumptions to simplify calculation:

$$
\begin{array}{ll}
\Theta_{i}=\left\{a_{1}^{i}, \ldots, a_{\left|\Theta_{i}\right|}^{i}\right\}, & a_{1}^{i}<\cdots<a_{\left|\Theta_{i}\right|}^{i} \in[1: v], \\
\bar{\Theta}_{i}=\left\{b_{1}^{i}, \ldots, b_{\left|\Theta_{i}\right|}^{i}\right\}, & b_{1}^{i}<\cdots<b_{\left|\Theta_{i}\right|}^{i} \in[1: \nu], \\
\Xi_{i}=\left\{c_{1}^{i}, \ldots, c_{\left|\Xi_{i}\right|}^{i}\right\}, & c_{1}^{i}<\cdots<c_{\left|\Xi_{i}\right|}^{i} \in[1: \kappa], \\
\bar{\Xi}_{i}=\left\{d_{1}^{i}, \ldots, d_{\left|\bar{\Xi}_{i}\right|}^{i}\right\}, & d_{1}^{i}<\cdots<d_{\left|\Xi_{i}\right|}^{i} \in[1: \kappa] .
\end{array}
$$

Then, under assumption (3), one has that

$$
\begin{aligned}
& x_{i}(\tau+1)=\left[x_{a_{1}^{i}}(\tau)\right] \sigma_{1}^{i}\left[x_{a_{2}^{i}}(\tau)\right] \sigma_{2}^{i}, \ldots, \sigma_{\left|\Theta_{i}\right|-1}^{i}\left[x_{a_{\left|\Theta_{i}\right|}^{i} \mid}(\tau)\right] \sigma_{\left|\Theta_{i}\right|}^{i}\left[\bar{x}_{b_{1}^{i}}(\tau)\right] \sigma_{\left|\Theta_{i}\right|+1}^{i} \\
& \cdot\left[\bar{x}_{b_{2}^{i}}(\tau)\right] \sigma_{\left|\Theta_{i}\right|+2}^{i}, \ldots, \sigma_{\left|\Theta_{i}\right|+\left|\bar{\Theta}_{i}\right|-1}^{i}\left[\bar{x}_{b_{\left|\bar{\Theta}_{i}\right|}^{i}}(\tau)\right] \sigma_{\left|\Theta_{i}\right|+\left|\bar{\Theta}_{i}\right|}^{i}\left[\vartheta_{c_{1}^{i}}(\tau)\right] \\
& \widehat{\sigma}_{1}^{i}\left[\vartheta_{c_{2}^{i}}(\tau)\right] \widehat{\sigma}_{2}^{i}, \ldots<\widehat{\sigma}_{\left|\Xi_{i}\right|-1}^{i}\left[\vartheta_{c_{\left|\Xi_{i}\right|}^{i}}(\tau)\right] \widehat{\sigma}_{\left|\Xi_{i}\right|}^{i}\left[\bar{\vartheta}_{d_{1}^{i}}(\tau)\right] \widehat{\sigma}_{\left|\Xi_{i}\right|+1}^{i}\left[\bar{\vartheta}_{d_{2}^{i}}(\tau)\right] \widehat{\sigma}_{\left|\Xi_{i}\right|+2}^{i}, \ldots, \widehat{\sigma}_{\left|\Xi_{i}\right|+\left|\bar{\Xi}_{i}\right|-1}^{i}\left[\bar{\vartheta}_{d_{\left|\bar{E}_{i}\right|}^{i}}(\tau)\right], \quad i \in[1: \mu] \text {, }
\end{aligned}
$$

where binary operators $\sigma_{j}^{i} \in\{\vee, \wedge\}, \quad j \in\left[1:\left|\Theta_{i}\right|+\left|\bar{\Theta}_{i}\right|\right]$, $i \in[1: \nu], \widehat{\sigma}_{j}^{i} \in\{\vee, \wedge\}, j \in\left[1:\left|\Xi_{i}\right|+\left|\bar{\Xi}_{i}\right|-1\right]$, and $i \in[1: \nu]$. Here, operators $\sigma_{j}^{i}, j \in\left[1:\left|\Theta_{i}\right|+\left|\bar{\Theta}_{i}\right|\right], \widehat{\sigma}_{j}^{i}$, and $j \in\left[1:\left|\Xi_{i}\right|+\right.$ $\left.\left|\bar{\Xi}_{i}\right|-1\right]$ are binary operators connecting the in-neighbor sets $\Theta_{i}, \bar{\Theta}_{i}, \Xi_{i}$, and $\bar{\Xi}_{i}, i \in[1: \nu]$.

Given binary operators $\sigma_{j} \in\{\vee, \wedge\}, j \in\left[1:\left|\Theta_{i}\right|+\left|\bar{\Theta}_{i}\right|\right]$, and $\widehat{\sigma}_{j} \in\{\vee, \wedge\}, j \in\left[1:\left|\Xi_{i}\right|+\left|\Xi_{i}\right|-1\right]$, one has that

$$
\begin{aligned}
x_{i}(\tau+1)= & \mathbf{O}_{i} \ltimes_{j=1}^{\left|\Theta_{i}\right|} x_{a_{i, j}}(\tau) \alpha_{j=1}^{\left|\bar{\Theta}_{i}\right|}\left(M_{n} \propto x_{b_{i, j}}(\tau)\right) \\
& \ltimes_{j=1}^{\left|\Xi_{i}\right|} \vartheta_{c_{i, j}}(\tau) \ltimes_{j=1}^{\left|\Xi_{i}\right|}\left(M_{n} \ltimes \vartheta_{d_{i, j}}(\tau)\right),
\end{aligned}
$$

where matrix $\mathbf{O}_{i}$ is uniquely calculated according to binary operators $\sigma_{j} \in\{\vee, \wedge\}, j \in\left[1:\left|\Theta_{i}\right|+\left|\bar{\Theta}_{i}\right|\right]$, and $\widehat{\sigma}_{j} \in\{\vee, \wedge\}$, $j \in\left[1:\left|\Xi_{i}\right|+\left|\bar{\Xi}_{i}\right|-1\right]$. Thus, there totally exist $2^{\left|\Theta_{i}\right|+\left|\bar{\Theta}_{i}\right|+\left|\Xi_{i}\right|+\left|\bar{\Xi}_{i}\right|-1}$ different kinds of structure matrices for matrices $\mathbf{O}_{i}, i \in[1: \mu]$. Given each $i \in[1: \mu]$, illustrate all the $2^{\left|\Theta_{i}\right|+\left|\bar{\Theta}_{i}\right|+\left|\Xi_{i}\right|+\left|\Xi_{i}\right|-1}$ kinds of matrices $\mathbf{O}_{i}$ in (5) by set $\Gamma_{i}$.
Denoting $x(\tau)=\ltimes_{i=1}^{v} x_{i}(\tau)$ and $\vartheta(\tau)=\ltimes_{i=1}^{\kappa} \vartheta_{i}(\tau)$, one can further calculate a unique matrix $\mathbf{T}_{i} \in \mathfrak{\Omega}_{2 \times 2^{v+\kappa}}, i \in[1: \mu]$, based on Lemma 1, satisfying

$$
\begin{aligned}
& \ltimes_{j=1}^{\left|\Theta_{i}\right|} x_{a_{i, j}}(\tau) \ltimes_{j=1}^{\left|\Theta_{i}\right|}\left(M_{n} \ltimes x_{b_{i, j}}(\tau)\right) \ltimes_{j=1}^{\left|\Xi_{i}\right|} \vartheta_{c_{i, j}}(\tau) \ltimes_{j=1}^{\left|\bar{\Xi}_{i}\right|}\left(M_{n} \ltimes \vartheta_{d_{i, j}}(\tau)\right) \\
& =\mathbf{T}_{i} \vartheta(\tau) x(\tau) .
\end{aligned}
$$

Thus, for $x_{1}, \ldots, x_{\mu}$, the following equation is obtained:

$$
x_{i}(\tau+1)=\mathbf{O}_{i} \mathbf{T}_{i} \vartheta(\tau) x(\tau) .
$$

Let $\mathbf{x}_{1}(\tau)=\ltimes_{i=1}^{\mu} x_{i}(\tau) \in \Delta_{2^{\mu}}$; it leads to a simplification illustration of (4) for nodes $x_{1}, \ldots, x_{\mu}$, using Khatri-Rao product,

$$
\mathbf{x}_{1}(\tau+1)=F_{1} \vartheta(\tau) x(\tau)
$$


where $F_{1} \in \Lambda_{1}: \triangleq\left\{*_{i=1}^{\mu}\left(\mathbf{O}_{i} \ltimes \mathbf{T}_{i}\right): \mathbf{O}_{i} \in \Gamma_{i}, i \in[1: \mu]\right\}$. Then, one has $\left|\Lambda_{1}\right|=2 \sum_{i=1}^{\mu}\left(\left|\Theta_{i}\right|+\left|\bar{\Theta}_{i}\right|+\left|\Xi_{i}\right|+\left|\Xi_{i}\right|\right)-\mu$. Denoting $r=\left|\Lambda_{1}\right|$, assume that $\Lambda_{1}=\left\{F_{1}^{1}, F_{1}^{2}, \ldots, F_{1}^{r}\right\}$, where matrices $F_{1}^{1}, F_{1}^{2}, \ldots, F_{1}^{r} \in \mathbf{\Omega}_{2^{\mu} \times 2^{*+\kappa}}$.

Then, let $\mathbf{x}_{2}(\tau)=\ltimes_{i=\mu+1}^{\nu} x_{i}(\tau) \in \Delta_{2^{\nu-\mu}}$; one can obtain algebraic form for the last $\nu-\mu$ nodes:

$$
\mathbf{x}_{2}(\tau+1)=F_{2} \vartheta(\tau) x(\tau)
$$

where $F_{2} \in \mathfrak{Q}_{2^{\nu-\mu} \times 2^{\nu+\kappa}}$ expresses the structure matrix determined by in-neighbor sets $\Theta_{i}, \bar{\Theta}_{i}, \Xi_{i}$, and $\bar{\Xi}_{i}, i \in[\mu+1: v]$, and its binary operators. Therefore, it leads to totally $s=$ $2^{(\nu-\mu)\left(2^{\nu+\kappa}\right)}$ cases for matrix $F_{2}$. Denote the set of all the $2^{(\nu-\mu)\left(2^{v+\kappa}\right)}$ different kinds of $F_{2}$ by $\Lambda_{2}$, that is, $F_{2} \in \Lambda_{2} \triangleq\left\{F_{2}^{1}, F_{2}^{2}, \ldots, F_{2}^{s}\right\}$, where $F_{2}^{1}, F_{2}^{2}, \ldots, F_{2}^{s} \in \mathfrak{Q}_{2^{v-\mu} \times 2^{\nu+\kappa}}$. Therefore, considering (2), one has that

$$
\left\{\begin{array}{l}
\mathbf{x}_{1}(\tau+1)=F_{1} \vartheta(\tau) x(\tau), \\
\mathbf{x}_{2}(\tau+1)=F_{2} \vartheta(\tau) x(\tau),
\end{array}\right.
$$

where

$$
F_{1} \in \Lambda_{1}=\left\{F_{1}^{1}, F_{1}^{2}, \ldots, F_{1}^{r}\right\}
$$

and

$F_{2} \in \Lambda_{2}=\left\{F_{2}^{1}, F_{2}^{2}, \ldots, F_{2}^{s}\right\}$. Multiplying (10) yields an algebraic form of (2),

$$
x(\tau+1)=F_{1} \vartheta(\tau) x(\tau) F_{2} \vartheta(\tau) x(\tau):=L \vartheta(\tau) x(\tau),
$$

where matrix $L$ is called the state transition matrix of system (2) choosing from a set $\Omega \triangleq\left\{F_{1} * F_{2}, F_{1} \in \Lambda_{1}, F_{2} \in \Lambda_{2}\right\}$. In addition, one can calculate that $|\Omega|=r s$ and represent all of the state transition matrices by $\Omega \triangleq\left\{L_{1}, L_{2}, \ldots, L_{r s}\right\}$, where $L_{1}, L_{2}, \ldots, L_{r s} \in \mathfrak{Q}_{2^{v} \times 2^{v+\kappa}}$. This implies that each matrix $L_{i}$, $i \in[1: r s]$, is one possible state transition matrix for system (2), where only the corresponding in-neighbor sets $\Theta_{i}, \bar{\Theta}_{i}, \Xi_{i}$, and $\bar{\Xi}_{i}, i \in[1: \mu]$, for those first $\mu$ nodes, are known.

\section{Main Results}

In this section, several types of controllability of (2) are presented, where only the corresponding in-neighbors of nodes $x_{1}, \ldots, x_{\mu}$, are known.

3.1. First Class of Structural Controllability. At the beginning, only the corresponding in-neighbor sets $\Theta_{i}, \bar{\Theta}_{i}, \Xi_{i}$, and $\bar{\Xi}_{i}$ for $x_{1}, \ldots, x_{\mu}$, are supposed to be known, the corresponding Boolean functions $\mathbf{g}_{i}, i \in[1: \mu]$, and both the in-neighbor sets $\Theta_{j}, \bar{\Theta}_{j}, \Xi_{j}$, and $\bar{\Xi}_{j}$ and the Boolean functions $\mathbf{g}_{j}$, $j \in[\mu+1: v]$, are unknown. Under these assumptions, reachability and controllability for (2) are firstly defined as follows.

Firstly, consider the first class structural reachability (FCSR) between two given states $x_{0}$ and $x_{d}$ under any Boolean functions $\mathbf{g}_{i}, i \in[1: v]$.

Definition 2. Consider system (2) and the neighbor sets $\Theta_{i}, \bar{\Theta}_{i}, \Xi_{i}$, and $\bar{\Xi}_{i}$ for $x_{1}, \ldots, x_{\mu}$, are available. The destination state $x_{d} \in \Delta_{2^{v}}$ achieves FCSR from the initial state $x_{0} \in \Delta_{2^{v}}$, if $x_{0}$ can be steered to $x_{d}$ for arbitrary Boolean functions $\mathbf{g}_{1}, \ldots, \mathbf{g}_{v}$. In addition, (2) achieves first class structural controllability (FCSC) if for any initial state $x_{0}$ and destination state $x_{d}, x_{d}$ achieves FCSR from $x_{0}$.

By considering $\Xi=\left\{L_{1}, L_{2}, \ldots, L_{r s}\right\}$, divide $L_{1}, \ldots, L_{r s}$, as follows: $L_{i}=\left[\operatorname{Blk}_{1}\left(L_{i}\right), \operatorname{Blk}_{2}\left(L_{i}\right), \ldots, \operatorname{Blk}_{2^{k}}\left(L_{i}\right)\right]$, and denote

$$
\mathrm{C}_{i}=\sum_{l=1}^{2^{v}}{ }_{\mathrm{B}}\left(M_{i}\right)^{(l)}, \quad \in \mathfrak{R}_{2^{v} \times 2^{v}}, i \in[1: r s],
$$

where $M_{i}=L_{i} \times 1_{2^{x}} \in \mathfrak{2}_{2^{v} \times 2^{v}}$, and matrix $C_{i}, i \in[1: r s]$, is the controllability matrix when state transition matrix of system (2) is $L_{i}$. Then, introduce the following Boolean matrix in order to issue FCSC,

$$
S_{1}=\mathrm{C}_{1} \circ \mathrm{C}_{2} \circ \cdots \circ \mathrm{C}_{r s} \in \mathfrak{\Omega}_{2^{v} \times 2^{v}},
$$

where "。" represents the well-known Hadamard matrix product. Here, name $S_{1}$ as FCSC matrix of (2) with partial inneighbor sets $\Theta_{i}, \bar{\Theta}_{i}, \Xi_{i}$, and $\bar{\Xi}_{i}$ for nodes $x_{i}$ available, $i \in[1: \mu]$.

By referring to matrix $S_{1}$ established in (13), the following criteria for FCSC are established.

Theorem 1. Consider system (2) with available sets $\Theta_{i}, \bar{\Theta}_{i}, \Xi_{i}$, and $\bar{\Xi}_{i}$ for nodes $x_{1}, \ldots, x_{\mu}$, then state $x_{d}=\delta_{2^{v}}^{\pi} \in \Delta_{2^{v}}\left(\pi \in\left[1: 2^{v}\right]\right)$ achieves FCSR from state $x_{0}=$ $\delta_{2^{v}}^{\eta} \in \Delta_{2^{v}}\left(\eta \in\left[1: 2^{\nu}\right]\right)$ if and only if the following condition $\left[S_{1}\right]_{\pi, \eta}=1$ holds.

Proof. According to the definition of Hadamard product "०," one can easily obtain that $\left[S_{1}\right]_{\pi, \eta}=1$ if and only if for each $i \in[1: r s], \quad\left[\mathrm{C}_{i}\right]_{\pi, \eta}=1$. For system $x(\tau+1)=L_{i} \vartheta(\tau) x(\tau), i \in[1: r s]$, according to [31], one has that $x_{d}=\delta_{2^{\nu}}^{\pi}$ is reachable from $x_{0}=\delta_{2^{\nu}}^{\eta}$ if and only if $\left[\mathrm{C}_{i}\right]_{\pi, \eta}=1$. Thus, by Definition 2, $x_{d}=\delta_{2^{v}}^{\pi}$ achieves FCSC from $x_{0}=\delta_{2^{n}}^{\eta}$ if and only if condition $\left[S_{1}\right]_{\pi, \eta}=1$ holds.

Then, FCSC for (2) with available partial in-neighbor sets $\Theta_{i}, \bar{\Theta}_{i}, \Xi_{i}$, and $\bar{\Xi}_{i}, i \in[1: \mu]$, is established.

Theorem 2. Given system (2) with available neighbor sets $\Theta_{i}, \bar{\Theta}_{i}, \Xi_{i}$, and $\bar{\Xi}_{i}$ for nodes $x_{1}, \ldots, x_{\mu}$, (2) achieves FCSC if and only if condition $S_{1}=1_{2^{v} \times 2^{v}}$ holds.

Proof. From Theorem 1, for any initial state $x_{0}$ and destination state $x_{d} \in \Delta_{2^{v}}, x_{d}$ achieves FCSR from $x_{0}$ if and only if $S_{1}=1_{2^{\nu} \times 2^{\nu}}$. Thus, according to Definition 2, system (2) achieves FCSC.

3.2. Second Class of Structural Controllability. Based on Definition 2, FCSR is introduced on reachability between the initial state $x_{0}$ and destination state $x_{d}$ for any Boolean functions $\mathbf{g}_{1}, \ldots, \mathbf{g}_{v}$. Hence, assume that state $x_{0}$ can be steered to state $x_{d}$ under certain possible Boolean mappings $\mathbf{g}_{i}, i \in[1: v]$, then the following second class of structural controllability is established. 
Definition 3. Given system (2) and the neighbor sets $\Theta_{i}, \bar{\Theta}_{i}, \Xi_{i}$, and $\bar{\Xi}_{i}$, for nodes $x_{1}, \ldots, x_{\mu}$, are available, the destination state $x_{d} \in \Delta_{2^{v}}$ achieves second class structural reachability (SCSR) from the initial state $x_{0} \in \Delta_{2^{v}}$, if there is certain Boolean functions $\mathbf{g}_{1}, \ldots, \mathbf{g}_{\nu}$ satisfying that $x_{d}$ can be reachable from $x_{0}$. In addition, (2) achieves SCSC if for any $x_{0}, x_{d} \in \Delta_{2^{v}}, x_{d}$ achieves second class structural controllability (SCSR) from $x_{0}$. define

By referring to established matrices $\mathrm{C}_{i}$ in (12), $i \in[1: r s]$,

$$
S_{2}=\sum_{i=1}^{r s}{ }_{\mathrm{B}} \mathrm{C}_{i}, \quad \in \mathfrak{\Omega}_{2^{v} \times 2^{v}} .
$$

We name $S_{2}$ as the SCSC matrix of (2) with available sets $\Theta_{i}, \bar{\Theta}_{i}, \Xi_{i}$, and $\bar{\Xi}_{i}$ for nodes $x_{i}, i \in[1: \mu]$. According to $S_{2}$ defined in (14), the following necessary and sufficient criteria are derived.

Theorem 3. Consider system (2) with available neighbor sets $\Theta_{i}, \bar{\Theta}_{i}, \Xi_{i}$, and $\bar{\Xi}_{i}$ for nodes $x_{1}, \ldots, x_{\mu}$, then state $x_{d}=\delta_{2^{v}}^{\pi} \in \Delta_{2^{v}}$ achieves SCSR from state $x_{0}=\delta_{2^{v}}^{\eta} \in \Delta_{2^{v}}$ if and only if condition $\left[S_{2}\right]_{\pi, \eta}=1$ holds.

Proof. $\left[S_{2}\right]_{\pi, \eta}=1$ if and only if there is a sequence of Boolean matrices $\mathrm{C}_{k}, k \in[1: r s]$, such that $\left[\mathrm{C}_{k}\right]_{\pi, \eta}=1$. For system $x(\tau+1)=L_{k} \vartheta(\tau) x(\tau)$, according to [31], one has that $x_{d}=\delta_{2^{v}}^{\pi}$ can be reachable from $x_{0}=\delta_{2^{\nu}}^{\eta}$ if and only if $\left[\mathrm{C}_{k}\right]_{\pi, \eta}=1$. Thus, by Definition 3, $x_{d}=\delta_{2^{v}}^{\pi^{2}}$ achieves SCSR from $x_{0}=\delta_{2^{v}}^{\eta}$ if and only if condition $\left[S_{2}\right]_{\pi, \eta}=1$ holds.

Theorem 4. Considering system (2), the neighbor sets $\Theta_{i}, \bar{\Theta}_{i}, \Xi_{i}$, and $\bar{\Xi}_{i}$ for nodes $x_{i}, i \in[1: \mu]$, are available, then (2) achieves SCSC if and only if condition $S_{2}=1_{2^{v} \times 2^{v}}$ holds.

Proof. From Theorem 3, for any two states $x_{0}, x_{d} \in \Delta_{2^{v}}$, state $x_{d}$ achieves SCSR from state $x_{0}$ if and only if condition $S_{2}=1_{2^{v} \times 2^{v}}$ holds. Thus, according to Definition 3, system (2) achieves SCSC.

In the above, controllability of (2) with known inneighbor sets $\Theta_{i}, \bar{\Theta}_{i}, \Xi_{i}$, and $\bar{\Xi}_{i}$ for nodes $x_{1}, \ldots, x_{\mu}$, under the situations of the arbitrariness and existence of logical functions $\mathbf{g}_{1}, \ldots, \mathbf{g}_{v}$, has been established, which are shown in Theorems 1 and 3. In the following, controllability of (2) under the situation of arbitrary logical functions $\mathbf{g}_{i}$, $i \in[1: \mu]$, and certain possible logical functions $\mathbf{g}_{j}$, $j \in[\mu+1: \nu]$, will be studied.

3.3. Third Class of Structural Controllability. In the following sequel, we consider the reachability between two given states $x_{0}$ and $x_{d}$ under any Boolean functions $\mathbf{g}_{i}, i \in[1: \mu]$, and certain possible logical functions $\mathbf{g}_{i}, i \in[\mu+1: \nu]$.

Definition 4. Considering system (2) and the neighbor sets $\Theta_{i}, \bar{\Theta}_{i}, \Xi_{i}$, and $\bar{\Xi}_{i}$, for nodes $x_{1}, \ldots, x_{\mu}$, are available, the destination state $x_{d} \in \Delta_{2^{v}}$ achieves third class structural reachability (TCSR) from the initial state $x_{0} \in \Delta_{2^{v}}$, if for any
Boolean functions $\mathbf{g}_{i}, i \in[1: \mu]$, there are certain Boolean mappings $\mathbf{g}_{j}, j \in[\mu+1: \nu]$ satisfying that $x_{d}$ can be reachable from $x_{0}$. In addition, (2) achieves third class structural controllability (TCSC), if for any $x_{0}, x_{d} \in \Delta_{2^{v}}, x_{d}$ achieves TCSR from $x_{0}$.

For convenience, denote the set of $L$ in (11) by $\Xi=$ $\left\{F_{1} * F_{2}, F_{1} \in \Lambda_{1}, F_{2} \in \Lambda_{2}\right\} \triangleq\left\{L^{1,1}, L^{1,2}, \ldots, L^{1, s}, L^{2,1}, L^{2,2}\right.$, $\left.\ldots, L^{2, s}, \ldots, L^{r, 1}, L^{r, 2}, \ldots, L^{r, s}\right\}$, where $L^{i, j}=F_{1}^{i} * F_{2}^{j}, \quad i \in$ [1: $r], j \in[1: s]$. Divide $L^{i, j}$ into $2^{\kappa}$ equal blocks as $L^{i, j}=\left[\mathrm{Blk}_{1} L^{i, j}, \mathrm{Blk}_{2} L^{i, j}, \ldots, \mathrm{Blk}_{2^{k}} L^{i, j}\right]$, and denote

$$
\mathrm{C}^{i, j}=\sum_{l=1}^{2^{v}}{ }_{\mathrm{B}}\left(M^{i, j}\right)^{(l)}, \quad \in \mathfrak{\Omega}_{2^{v} \times 2^{v}}, i \in[1: r], j \in[1: s],
$$

where $M^{i, j}=L^{i, j}<i$ mes $1_{2^{k}} \in \mathbb{2}_{2^{\nu} \times 2^{\nu}}$, and matrix $C^{i, j}$ is the corresponding controllability matrix when state transition matrix of system (2) is $L^{i, j}$. In addition, introduce the following Boolean matrix in order to issue TCSC,

$$
S_{3}=T^{1} \circ T^{2} \circ \cdots \circ T^{r}, \quad \in \mathfrak{R}_{2^{v} \times 2^{v}},
$$

where $T^{i}=\sum_{j=1 \mathrm{~B}}^{s} \mathrm{C}^{i, j}, \in \mathfrak{R}_{2^{\nu} \times 2^{\nu}}, i \in[1: r]$. We name $S_{3}$ as TCSC matrix of (2) with available sets $\Theta_{i}, \bar{\Theta}_{i}, \Xi_{i}$, and $\bar{\Xi}_{i}$ for $x_{1}, \ldots, x_{\mu}$.

By referring to matrix $S_{3}$ defined in (16), the following criteria for TCSC are established.

Theorem 5. Given system (2), for $i \in[1: \mu]$, the neighbor sets $\Theta_{i}, \bar{\Theta}_{i}, \Xi_{i}$, and $\bar{\Xi}_{i}$ for nodes $x_{i}$ are available, then state $x_{d}=$ $\delta_{2^{v}}^{\pi} \in \Delta_{2^{v}}$ achieves LCSR from state $x_{0}=\delta_{2^{v}}^{\eta} \in \Delta_{2^{v}}$ if and only if condition $\left[S_{3}\right]_{\pi, \eta}=1$ holds.

Proof. $\left[S_{3}\right]_{\pi, \eta}=1$ if and only if $\left[T^{i}\right]_{\pi, \eta}=1, i \in[1: r]$, and there is a sequence of Boolean matrices $C^{i, k}, k \in[1: s]$, such that $\left[\mathrm{C}^{i, k}\right]_{\pi, \eta}=1$ for every $\left[T^{i}\right]$. For system $x(\tau+1)=L^{i, k} \vartheta(\tau) x(\tau)$, according to [31], one has that $x_{d}=$ $\delta_{2^{v}}^{\pi}$ is reachable from $x_{0}=\delta_{2^{v}}^{\eta}$ if and only if $\left[\mathrm{C}^{i, k}\right]_{\pi, \eta}=1$. Thus, by Definition 4, state $x_{d}=\delta_{2^{v}}^{\pi}$ achieves TCSR from state $x_{0}=\delta_{2^{\nu}}^{\eta}$ if and only if condition $\left[S_{3}\right]_{\pi, \eta}=1$ holds.

Theorem 6. Given system (2), the neighbor sets $\Theta_{i}, \bar{\Theta}_{i}, \Xi_{i}$, and $\bar{\Xi}_{i}$ for nodes $x_{i}, i \in[1: \mu]$, are available, then (2) achieves TCSC if and only if condition $S_{3}=1_{2^{y} \times 2^{y}}$ holds.

Proof. From Theorem 5, for any two states $x_{0}, x_{d} \in \Delta_{2^{v}}$, state $x_{d}$ achieves TCSR from state $x_{0}$ if and only if condition $S_{3}=1_{2^{v} \times 2^{v}}$ holds. Thus, according to Definition 4, system (2) achieves TCSC.

Remark 1. More strictly, if for $\mathbf{g}_{j}, j \in[\mu+1: \nu]$, logical functions are the same, then for any functions $\mathbf{g}_{i}, i \in[1: \mu]$, we define the TCSC matrix as $S_{3}=\sum_{j=1 \mathrm{~B}}^{s} \widetilde{T}^{j}, \in \mathfrak{\Omega}_{2^{v} \times 2^{v}}$, where $\widetilde{T}^{j}=C^{1, j} \circ C^{2, j} \circ \ldots \circ C^{r, j} \in \mathfrak{R}_{2^{v} \times 2^{v}}, j \in[1: s]$.

3.4. Last Class of Structural Controllability. Based on Definition 4 , TCSR is introduced on reachability between the initial state $x_{0}$ and destination state $x_{d}$ for any Boolean 
functions $\mathbf{g}_{i}, i \in[1: \mu]$ and certain Boolean functions $\mathbf{g}_{i}$, $i \in[\mu+1: v]$. Thus, if destination state $x_{d}$ is reachable from the initial state $x_{0}$ under certain Boolean functions $\mathbf{g}_{i}$, $i \in[1: \mu]$ and any Boolean functions $\mathbf{g}_{i}, i \in[\mu+1: \nu]$, the following last class of structural controllability is established.

Definition 5. Considering system (2), for $i \in[1: \mu]$, the neighbor sets $\Theta_{i}, \bar{\Theta}_{i}, \Xi_{i}$, and $\bar{\Xi}_{i}$ for nodes $x_{i}$ are available. The destination state $x_{d} \in \Delta_{2^{v}}$ achieves last class structural reachability (LCSR) from initial state $x_{0} \in \Delta_{2^{v}}$, if there is certain Boolean functions $\mathbf{g}_{1}, \ldots, \mathbf{g}_{p}$ satisfying that $x_{d}$ can be reachable from $x_{0}$ for any Boolean functions $\mathbf{g}_{i}, i \in[\mu+1: \nu]$. Moreover, (2) achieves last class structural controllability (LCSC) if for any $x_{0}, x_{d} \in \Delta_{2^{v}}$, state $x_{d}$ achieves LCSR from state $x_{0}$.

By referring to matrix $C^{i, j}$ established in (15), $i \in[1: r], j \in[1: s]$, define

$$
S_{4}=\sum_{i=1}^{r} \widehat{B}^{i} \in \mathfrak{R}_{2^{\nu} \times 2^{\nu}},
$$

where $\widehat{T}^{i}=C^{i, 1} \circ C^{i, 2} \circ \ldots \circ C^{i, s}, i \in[1: r]$. We call $S_{4}$ as LCSC matrix of (2) with available sets $\Theta_{i}, \bar{\Theta}_{i}, \Xi_{i}$, and $\bar{\Xi}_{i}$ for nodes $x_{1}, \ldots, x_{\mu}$.

By referring to matrix $S_{4}$ defined in (17), the following criteria for LCSC are obtained.

Theorem 7. Given system (2), for $i \in[1: \mu]$, the neighbor sets $\Theta_{i}, \bar{\Theta}_{i}, \Xi_{i}$, and $\bar{\Xi}_{i}$ for nodes $x_{i}$ are available, then the destination state $x_{d}=\delta_{2^{v}}^{\pi} \in \Delta_{2^{v}}$ achieves LCSR from the initial state $x_{0}=\delta_{2^{v}}^{\eta} \in \Delta_{2^{v}}$ if and only if condition $\left[S_{4}\right]_{\pi, \eta}=1$ holds.

Proof. $\left[S_{4}\right]_{\pi, \eta}=1$ if and only if there exists a sequence of matrices $\widehat{T}^{k}, k \in[1: r]$, such that $\left[\widehat{T}^{k}\right]_{\pi, \eta}=1$, and for every matrix $\quad C^{k, j}, j \in[1: s], \quad\left[C^{k, j}\right]_{\pi, \eta}=1$. For system $x(\tau+1)=L^{k, j} \vartheta(\tau) x(\tau)$, according to [31], one has that $x_{d}=$ $\delta_{2^{v}}^{\pi}$ is reachable from $x_{0}=\delta_{2^{v}}^{\eta}$ if and only if $\left[C^{k, j}\right]_{\pi, \eta}=1$. Thus, by Definition 5, $x_{d}=\delta_{2^{v}}^{\pi}$ achieves LCSR from $x_{0}=\delta_{2^{v}}^{\eta}$ if and only if condition $\left[S_{4}\right]_{\pi, \eta}=1$ holds.

Theorem 8. Given system (2), for $i \in[1: \mu]$, the neighbor sets $\Theta_{i}, \bar{\Theta}_{i}, \Xi_{i}$, and $\bar{\Xi}_{i}$ for nodes $x_{i}$ are available, then (2) achieves LCSC if and only if condition $S_{4}=1_{2^{v} \times 2^{v}}$ holds.

Proof. From Theorem 7, for any initial state $x_{0}$ and destination state $x_{d} \in \Delta_{2^{v}}$, state $x_{d}$ achieves LCSR from state $x_{0}$ if and only if condition $S_{4}=1_{2^{v} \times 2^{v}}$ holds. Thus, according to Definition 5, system (2) achieves LCSC.

Remark 2. In this paper, four types of SC of BCNs with partial information available have been studied, where one only knows part of messages on nodes' connections. Among the abovementioned four different kinds of structural controllability, the relationships are shown as follows: FCSC implies TCSC and LCSC; the third and the last class of SC both imply SCSC. In addition, FCSC is the most conservative definition and all other types of SC can be implied by FCSC, and the diagram describing the detailed relationships among different kinds of SC is shown as Figure 1.

Remark 3. By comparing with the recent theoretical results $[39,40]$, the established theoretical results in this paper are more general. If $p=n$, that is, one knows all the inneighbors of (2), then the results in [40] can be directly obtained from FCSC in this paper. Moreover, if $p=n$, FCSC in this paper implies common controllability [39], which means that system (2) is always controllable no matter which functions are chosen. Thus, the study of these four cases of SC of BCNs with partial information available is a simple extension of common controllability, which provides a new insight for further studying and reducing the high computational complexity of BCNs.

\section{Simulations}

Here, simulations on a Boolean model of the lactose operon in Escherichia coli are given to illustrate the obtained results.

Example 1. Firstly, a lactose operon model in Escherichia coli modeled by a BCN is considered [42]:

$$
\begin{aligned}
& w_{1}(\tau+1)=\left(\xi_{2}(\tau) \vee w_{3}(\tau)\right) \wedge \bar{\xi}_{1}(\tau), \\
& w_{2}(\tau+1)=w_{1}(\tau), \\
& w_{3}(\tau+1)=\left[\left(\xi_{2}(\tau) \wedge w_{2}(\tau)\right) \vee\left(\bar{w}_{2}(\tau) \wedge w_{3}(\tau)\right)\right] \wedge \bar{\xi}_{1}(\tau),
\end{aligned}
$$

where $w_{1} \in \mathfrak{D}$ denotes the biological structure of mRNA, $w_{2} \in \mathfrak{D}$ denotes the biological structure of lac $Z$ polypeptide, $w_{3} \in \mathfrak{D}$ denotes the intracellular lactose, $\xi_{1} \in \mathfrak{D}$ denotes the external glucose, and $\xi_{2} \in \mathfrak{D}$ denotes the external lactose.

Using STP tool, the corresponding algebraic form of system (18) is given as follows: $w(\tau+1)=L u(\tau) w(\tau)$, where $L=\delta_{8}[6,6,6,6,8,8,8,8,6,6,6,6,8,8,8,8,1,1,1,2,3,3,3,4$, $2,6,1,6,4,8,3,8]$. Then, split matrix $L$ into four blocks and obtain its controllability matrix $C=\sum_{l=1 \mathrm{~B}}^{8}(M)^{(l)} \in \mathfrak{B}_{8 \times 8}$, where $M=L \ltimes 1_{4} \in \mathfrak{B}_{8 \times 8}$. After a straightforward calculation, one has that $C=\left[\delta_{8}^{1}+\delta_{8}^{2}+\delta_{8}^{3}+\delta_{8}^{4}+\delta_{8}^{6}+\delta_{8}^{8}, \delta_{8}^{1}+\delta_{8}^{2}+\right.$ $\left.\delta_{8}^{3}+\delta_{8}^{4}+\delta_{8}^{6}+\delta_{8}^{8}, \ldots, \delta_{8}^{1}+\delta_{8}^{2}+\delta_{8}^{3}+\delta_{8}^{4}+\delta_{8}^{6}+\delta_{8}^{8}\right] \in \mathfrak{B}_{8 \times 8}$. By referring to [31], one can conclude that system (18) is not controllable.

Suppose that only in-neighbors for nodes $w_{1}$ and $w_{2}$ are given, but the detailed logical functions are unknown. Thus, the following system is considered:

$$
\begin{aligned}
& w_{1}(\tau+1)=f_{1}\left(w_{3}(\tau), \xi_{2}(\tau), \bar{\xi}_{1}(\tau)\right), \\
& w_{2}(\tau+1)=f_{2}\left(w_{1}(\tau)\right), \\
& w_{3}(\tau+1)=f_{3}\left(\left[w_{j}(\tau)\right]_{j \in \Theta_{3}},\left[\bar{w}_{j}(\tau)\right]_{j \in \bar{\Theta}_{3}},\left[\xi_{j}(\tau)\right]_{j \in \Xi_{3}},[\bar{\xi}(\tau)]_{j \in \bar{\Xi}_{3}}\right),
\end{aligned}
$$

where $\Theta_{3}$ and $\Xi_{3}\left(\bar{\Theta}_{3}\right.$ and $\left.\bar{\Xi}_{3}\right)$ express the unavailable sets of the activating indegrees (inhibiting indegrees) of node $w_{3}$.

Based on equations (5)-(9), a simple equivalent algebraic illustration of (19) can be derived: 


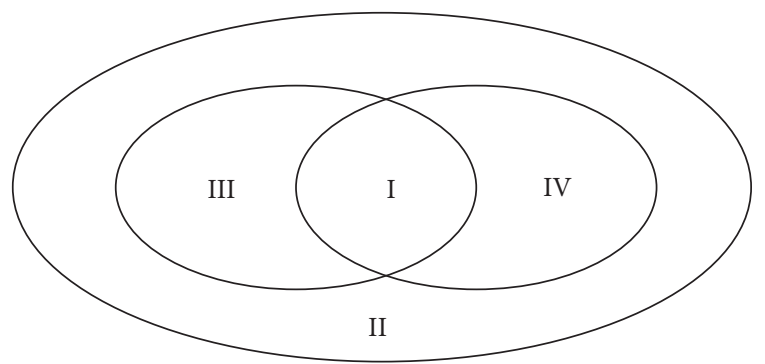

FIgURE 1: The implication relationships among different types of SC (I, II, III, and IV represent abbreviations of FCSC, SCSC, TCSC, and LCSC).

$$
\begin{aligned}
\mathbf{x}_{1}(\tau+1) & =F_{1} \xi(\tau) w(\tau), \\
\mathbf{w}_{2}(\tau+1) & =F_{2} \xi(\tau) w(\tau),
\end{aligned}
$$

where $\quad \mathbf{w}_{1}=w_{1} w_{2}, \quad \mathbf{w}_{2}=w_{3}, \quad \xi=\xi_{1} \xi_{2}, \quad F_{1} \in \Lambda_{1}=$ $\left\{F_{1}^{1}, F_{1}^{2}, F_{1}^{3}, F_{1}^{4}\right\}$, matrix $F_{2} \in \Lambda_{2}=\left\{F_{2}^{1}, \ldots, F_{2}^{2^{32}}\right\}$, and the cardinality of $\left|\Lambda_{2}\right|$ is $2^{32}$. Here, $F_{1}^{1}=\delta_{4}[3,3,3,3,4,4,4,4$, $3,3,3,3,4,4,4,4,1,3,1,3,2,4,2,4,3,3,3,3,4,4,4,4], \quad F_{1}^{2}=$ $\delta_{4}[3,3,3,3,4,4,4,4,3,3,3,3,4,4,4,4,1,1,1,1,2,2,2,2,1,3$, $1,3,2,4,2,4], F_{1}^{3}=\delta_{4}[1,3,1,3,2,4,2,4,3,3,3,3,4,4,4,4,1$, $1,1,1,2,2,2,2,1,1,1,1,2,2,2,2]$, and $F_{1}^{4}=\delta_{4}[1,1,1,1,2,2$, $2,2,1,3,1,3,2,4,2,4,1,1,1,1,2,2,2,2,1,1,1,1,2,2,2,2]$.

Thus, one can obtain its equivalent augmented system of (19): $\quad w(\tau+1)=L \xi(\tau) w(\tau)$, where matrix $L \in$ $\Omega=\left\{F_{1} * F_{2}, F_{1} \in \Lambda_{1}, F_{2} \in \Lambda_{2}\right\}=\left\{L_{1}, L_{2}, \ldots, L_{2^{34}}\right\}$. Due to the high computational complexity of the set $\Xi$, the detailed information for matrices $L_{1}, L_{2}, \ldots, L_{2^{34}}$ is omitted.

A straightforward computation shows that $S_{1} \neq$ $1_{8 \times 8}, S_{2}=1_{8 \times 8}, S_{3}=1_{8 \times 8}, S_{4} \neq 1_{8 \times 8}$. By referring to Theorems $1,3,5$, and 7, system (19) achieves SCSC and TCSC, while it cannot achieve FCSC or LCSC.

When $F_{2}=\delta_{2}[2,2,2,2,2,1,2,2,1,1,1,2,1,2,1,1,2,1,1$, $2,1,2,2,2,2,2,1,1,1,2,2,2]$, if $F_{1}=F_{1}^{1}$, then $L=\delta_{8}[6$, $6,6,6,8,7,8,8,5,5,5,6,7,8,7,7,2,5,1,6,3,8,4,8,6,6,5,5$, $7,8,8,8], C=1_{8 \times 8} ;$ if $F_{1}=F_{1}^{2}$, then $L=\delta_{8}[6,6,6,6,8$, $7,8,8,5,5,5,6,7,8,7,7,2,1,1,2,3,4,4,4,2,6,1,5,3,8,4,8]$, $C=1_{8 \times 8} ; \quad$ if $\quad F_{1}=F_{1}^{3}$, then $L=\delta_{8}[2,6,2,6,4,7,4,8,5,5$, $5,6,7,8,7,7,2,1,1,2,3,4,4,4,2,2,1,1,3,4,4,4], C=1_{8 \times 8}$; if $F_{1}=F_{1}^{4}$, then $L=\delta_{8}[2,2,2,2,4,3,4,4,1,5,1,6,3,8,3,7,2$, $1,1,2,3,4,4,4,2,2,1,1,3,4,4,4], \quad C=1_{8 \times 8}$. Thus, $S_{2}=$ $S_{3}=1_{8 \times 8}$. By referring to Theorems 3 and 5 , system (19) achieves SCSC and TCSC. This implies that, for any $w_{0}, w_{d} \in \Delta_{2^{v}}$, and any logical functions $f_{1}, f_{2}$, there is a logical functions $f_{3}$ satisfying that state $x_{d}$ can be reached from initial state $w_{0}$. According to Definitions 3 and 4, one can conclude that system (19) achieves SCSC and TCSC, respectively.

When $\quad F_{2}=\delta_{2}[2,2,2,2,2,2,2,2,2,2,2,2,2,2,2,2,1$, $1,1,2,1,1,1,2,2,2,1,2,2,2,1,2]$, that is, $w_{3}(\tau+1)=$ $\bar{\xi}_{1}(\tau) \wedge\left[\left(w_{2}(\tau) \wedge \xi_{2}(\tau)\right) \vee\left(w_{3}(\tau) \wedge \bar{w}_{2}(\tau)\right)\right]$, if $F_{1}=F_{1}^{1}$, then $L=\delta_{8}[6,6,6,6,8,8,8,8,6,6,6,6,8,8,8,8,1,5,1,6,3,7,3,8$, $6,6,5,6,8,8,7,8], C \neq 1_{8 \times 8} ;$ if $F_{1}=F_{1}^{2}$, then $L=\delta_{8}[6,6$, $6,6,8,8,8,8,6,6,6,6,8,8,8,8,1,1,1,2,3,3,3,4,2,6,1,6,4$, $8,3,8], \quad C \neq 1_{8 \times 8} ; \quad$ if $F_{1}=F_{1}^{3}$, then $L=\delta_{8}[2,6,2,6,4,8$, $4,8,6,6,6,6,8,8,8,8,1,1,1,2,3,3,3,4,2,2,1,2,4,4,3,4]$,
$C \neq 1_{8 \times 8} ; \quad$ if $\quad F_{1}=F_{1}^{4}$, then $\quad L=\delta_{8}[2,2,2,2,4,4,4,4,2$, $6,2,6,4,8,4,8,1,1,1,2,3,3,3,4,2,2,1,2,4,4,3,4], C \neq 1_{8 \times 8}$. Thus, $S_{4} \neq 1_{8 \times 8}$. According to Theorem 7 , system (19) cannot achieve LCSC. In this case, there exists a logical function $f_{3}$, such that system (19) is not controllable for any logical functions $f_{1}, f_{2}$. By Definition 5, system (19) cannot achieve LCSC.

\section{Conclusion}

In this paper, SC of BCNs has been systematically addressed, where only partial information of nodes' connections is available. By referring to its equivalent algebraic representation of BCNs, four kinds of SC have been established, based on different issues of nodes' connections. Then, certain criteria for different types of SC have been proposed, by defining its corresponding SC matrix. Finally, a Boolean model of the lactose operon in Escherichia coli has been simulated to show the effectiveness of the main theoretical results. One interesting research issue for future works includes SC of probabilistic BCNs with time-variant information of nodes' connections.

\section{Data Availability}

No data were used to support this study.

\section{Disclosure}

This paper was presented in part at the 11th International Conference on Information Science and Technology (ICIST), Chengdu, China, May 21-23, 2021.

\section{Conflicts of Interest}

The authors declare that they have no conflicts of interest.

\section{Acknowledgments}

This work was jointly supported by the National Natural Science Foundation of China (61903339), the National Training Programs of Innovation and Entrepreneurship (202010345009), the Guangdong Basic and Applied Basic Research Foundation (2019A1515110234), and the Shenzhen Science and Technology Program (RCBS20200714114921371).

\section{References}

[1] S. Kauffman, C. Peterson, B. Samuelsson, and C. Troein, "Random Boolean network models and the yeast transcriptional network," Proceedings of the National Academy of Sciences, vol. 100, no. 25, pp. 14796-14799, 2003.

[2] Z. Zhang, T. Leifeld, and P. Zhang, "Reduced-order observer design for Boolean control networks," IEEE Transactions on Automatic Control, vol. 65, no. 1, pp. 434-441, 2020.

[3] J. Zhong, Z. Yu, Y. Li, and J. Lu, "State estimation for probabilistic Boolean networks via outputs observation," IEEE Transactions on Neural Networks and Learning Systems, pp. 1-13, 2021. 
[4] J. Zhong, B. Li, Y. Liu, J. Lu, and W. Gui, "Steady-state design of large-dimensional Boolean networks," IEEE Transactions on Neural Networks and Learning Systems, vol. 32, no. 3, pp. 1149-1161, 2021.

[5] M. Meng, J. Lam, J. Feng, and K. Cheung, "Stability and guaranteed cost analysis of time-triggered Boolean networks," IEEE Transactions on Neural Networks and Learning Systems, vol. 29, no. 8, pp. 3893-3899, 2018.

[6] S. Zhu, J. Lu, and Y. Liu, "Asymptotical stability of probabilistic Boolean networks with state delays," IEEE Transactions on Automatic Control, vol. 65, no. 4, pp. 1779-1784, 2020.

[7] Y. Wu, Y. Guo, and M. Toyoda, "Policy iteration approach to the infinite horizon average optimal control of probabilistic Boolean networks," IEEE Transactions on Neural Networks and Learning Systems, pp. 1-15, 2020.

[8] R. Liu, J. Lu, W. X. Zheng, and J. Kurths, "Output feedback control for set stabilization of Boolean control networks," IEEE Transactions on Neural Networks and Learning Systems, vol. 31, no. 6, pp. 2129-2139, 2020.

[9] Y. Liu, L. Sun, J. Lu, and J. Liang, "Feedback controller design for the synchronization of Boolean control networks," IEEE Transactions on Neural Networks and Learning Systems, vol. 27, no. 9, pp. 1991-1996, 2016.

[10] G. Zhao, Y. Wang, and H. Li, "A matrix approach to the modeling and analysis of networked evolutionary games with time delays," IEEE/CAA Journal of Automatica Sinica, vol. 5, no. 4, pp. 818-826, 2018.

[11] F. Li and Y. Tang, "Pinning controllability for a Boolean network with arbitrary disturbance inputs," IEEE Transactions on Cybernetics, vol. 51, no. 6, pp. 3338-3347, 2021.

[12] M. Xu, Y. Liu, J. Lou, Z.-G. Wu, and J. Zhong, "Set stabilization of probabilistic Boolean control networks: a sampleddata control approach," IEEE Transactions on Cybernetics, vol. 50, no. 8, pp. 3816-3823, 2020.

[13] H. Chen and J. Liang, "Local synchronization of interconnected Boolean networks with stochastic disturbances," IEEE Transactions on Neural Networks and Learning Systems, vol. 31, no. 2, pp. 452-463, 2020.

[14] J. Zhong, Y. Liu, J. Lu, and W. Gui, "Pinning control for stabilization of Boolean networks under knock-out perturbation," IEEE Transactions on Automatic Control, p. 1, 2021.

[15] L. Lin, J. Zhong, S. Zhu, and J. Lu, "Sampled-data general partial synchronization of Boolean control networks," Journal of the Franklin Institute, 2020.

[16] S. Zhu, J. Lu, Y. Lou, and Y. Liu, "Induced-equations-based stability analysis and stabilization of Markovian jump Boolean networks," IEEE Transactions on Automatic Control, p. 1, 2020.

[17] S. Zhu, J. Lu, L. Lin, and Y. Liu, "Minimum-time and minimum-triggering control for the observability of stochastic Boolean networks," IEEE Transactions on Automatic Control, p. $1,2021$.

[18] L. Sun, W.-k. Ching, and J. Lu, "Stabilization of aperiodic sampled-data Boolean control networks: a delay approach," IEEE Transactions on Automatic Control, p. 1, 2021.

[19] D. Cheng, H. Qi, and Z. Li, "Analysis and control of Boolean networks: a semi-tensor product approach," in Proceedings of the 2009 7th Asian Control Conference, Hong Kong, China, August 2009.

[20] D. Laschov and M. Margaliot, "Controllability of Boolean control networks via the Perron-Frobenius theory," Automatica, vol. 48, no. 6, pp. 1218-1223, 2012.

[21] J. Zhong, B.-w. Li, Y. Liu, and W.-h. Gui, "Output feedback stabilizer design of Boolean networks based on network structure," Frontiers of Information Technology \& Electronic Engineering, vol. 21, no. 2, pp. 247-259, 2019.

[22] H. Li, S. Wang, X. Li, and G. Zhao, "Perturbation analysis for controllability of logical control networks," SIAM Journal on Control and Optimization, vol. 58, no. 6, pp. 3632-3657, 2020.

[23] J. Lu, H. Li, Y. Liu, and F. Li, "Survey on semi-tensor product method with its applications in logical networks and other finite-valued systems," IET Control Theory \& Applications, vol. 11, no. 13, pp. 2040-2047, 2017.

[24] Y. Wu and T. Shen, "Policy iteration approach to control residual gas fraction in IC engines under the framework of stochastic logical dynamics," IEEE Transactions on Control Systems Technology, vol. 25, no. 3, pp. 1100-1107, 2017.

[25] X. Li, X. Yang, and T. Huang, "Persistence of delayed cooperative models: impulsive control method," Applied Mathematics and Computation, vol. 342, pp. 130-146, 2019.

[26] X. Li, J. Shen, H. Akca, and R. Rakkiyappan, "LMI-based stability for singularly perturbed nonlinear impulsive differential systems with delays of small parameter," Applied Mathematics and Computation, vol. 250, pp. 798-804, 2015.

[27] X. Li, J. Shen, and R. Rakkiyappan, "Persistent impulsive effects on stability of functional differential equations with finite or infinite delay," Applied Mathematics and Computation, vol. 329, pp. 14-22, 2018.

[28] T. Akutsu, M. Hayashida, W.-K. Ching, and M. K. Ng, "Control of Boolean networks: hardness results and algorithms for tree structured networks," Journal of Theoretical Biology, vol. 244, no. 4, pp. 670-679, 2007.

[29] D. Cheng, H. Qi, Z. Li, and J. B. Liu, "Stability and stabilization of Boolean networks," International Journal of Robust and Nonlinear Control, vol. 21, no. 2, pp. 134-156, 2011.

[30] E. Fornasini and M. E. Valcher, "On the periodic trajectories of Boolean control networks," Automatica, vol. 49, no. 5, pp. 1506-1509, 2013.

[31] Y. Zhao, H. Qi, and D. Cheng, "Input-state incidence matrix of Boolean control networks and its applications," Systems \& Control Letters, vol. 59, no. 12, pp. 767-774, 2010.

[32] F. Li, "Pinning control design for the stabilization of Boolean networks," IEEE Transactions on Neural Networks and Learning Systems, vol. 27, no. 7, pp. 1585-1590, 2015.

[33] J. Lu, J. Zhong, C. Huang, and J. Cao, "On pinning controllability of Boolean control networks," IEEE Transactions on Automatic Control, vol. 61, no. 6, pp. 1658-1663, 2016.

[34] F. Li, "Robust stabilization for a logical system," IEEE Transactions on Control Systems Technology, vol. 25, no. 6, pp. 2176-2184, 2017.

[35] E. Sontag, A. Veliz-Cuba, R. Laubenbacher, and A. S. Jarrah, "The effect of negative feedback loops on the dynamics of Boolean networks," Biophysical Journal, vol. 95, no. 2, pp. 518-526, 2008.

[36] C. Campbell and R. Albert, "Stabilization of perturbed Boolean network attractors through compensatory interactions," BMC Systems Biology, vol. 8, no. 1, p. 53, 2014.

[37] E. Weiss, M. Margaliot, and G. Even, "Minimal controllability of conjunctive Boolean networks is NP-complete," Automatica, vol. 92, pp. 56-62, 2018.

[38] S.-i. Azuma, T. Yoshida, and T. Sugie, "Structural oscillatority analysis of Boolean networks," IEEE Transactions on Control of Network Systems, vol. 6, no. 2, pp. 464-473, 2019.

[39] Z. Gao, X. Chen, and T. Basar, "Controllability of conjunctive Boolean networks with application to gene regulation," IEEE Transactions on Control of Network Systems, vol. 5, no. 2, pp. 770-781, 2018. 
[40] S. Liang, H. Li, and S. Wang, "Structural controllability of Boolean control networks with an unknown function structure," Science China Information Sciences, vol. 63, no. 11, Article ID 219203, 2020.

[41] S. Tong, J. Zhong, and B. Li, "Structural controllability of Boolean control networks with known nodes coupling relationships," in Proceedings of the 2021 11th International Conference on Information Science and Technology, pp. 370374, Chengdu, China, May 2021.

[42] R. Robeva and T. Hodge, Mathematical Concepts and Methods in Modern Biology: Using Modern Discrete Models, Academic Press, Cambridge, MA, USA, 2003. 